\title{
Effect of Low Dose Oral Contraceptive Pill on Coagulation Status in Women with Normal and Low Body Mass Index
}

\author{
SB KASEM ${ }^{\mathrm{a}}, \mathrm{TACHOUDHURY}^{\mathrm{b}}, \mathrm{SARAZZAQUE}^{\mathrm{c}}, \mathrm{F} \mathrm{BEGUM}^{\mathrm{d}}$, RADIBA $^{\mathrm{e}}, \mathrm{S}^{\mathrm{N}}$ ANIKA $^{\mathrm{f}}$
}

\begin{abstract}
Summary:
Background: Oral contraceptive pill (OCP) is widely used by millions of women of various socioeconomic conditions. Use of low dose oestrogen and newer progesterone in OCP now associated with less thromboembolic and cardiovascular risk. The safety aspects of these pills had not been investigated in details in malnourished women of developing countries. In the present study the effects of the pillson coagulation status have been studied.
\end{abstract}

Objective: To explore the effect of the most widely used low dose OCP (Shukhi) on coagulation status of Bangladeshi women with normal and low BMI(Body Mass Index).

Materials and Methods: The study population group $(n=29)$ comprised of women with normal BMI and the underweight group $(n=11, B M I<18.5)$. Both groups use low dose OCP $(30 \mu \mathrm{g}$ ethinyl estradiol and 150 $\mathrm{\mu g}$ levonorgestrel) for 6-60 months.

The coagulation status were assessed as follows: Plasma Fibrinogen, Prothrombin time and Platlete aggregation and Anticoagulation statuseg: AntithrombinIII (ATIII).

Result:Coagulation status showed no significant difference in platelet aggregation between the groups. Plasma fibrinogen

Introduction:

OCP (combined) are very important component of family planning program. It is one of the effective methods of

a. Dr.Setara Binte Kasem, Assistant Professor (Gynae\& obstetrics), Sir Salimullah Medical College.

b. Prof. TA Chowdhury, Professor \& Head of the Dept of Gynae and Obstetrics, Ibrahim Medical College, Dhaka.

c. Dr. Shaikh Abdur Razzaque, Associate Professor (Paediatric Cardiology), National Institute of Cardiovascular diseases.

d. Dr. Ferdousi Begum, Professor (Gynae\&Obs), Ibrahim Medical College, Dhaka.

e. Dr. RaisaAdiba, Resident surgeon, Holly Family Red Crescent Medical College.

f. Dr. Selma Anika, Third year student, Bangladesh Medical College.

Address of Correspondence: Dr. Setara Binte Kasem, Assistant Professor (Gynae \& Obstetrics), Sir Salimullah Medical College. Mob-01552314895,Email-dr.setaraa@gmail.com.

Received: 6 May 2015

Accepted: 21 Sept. 2016 median value $(450 \mathrm{mg} / \mathrm{dl})$ just exceeded the upper limit of reference range (normal range: $200-400 \mathrm{mg} / \mathrm{dl}$ ) in normal BMI. In contrast, the corresponding value in the low BMI (318mg/ dl) group was almost at the middle of the reference range. $A$ significantly prolonged prothrombin time (13.80 seconds) was found in the low BMI group ( $p=0.058)$; values were still within the reference range (10-14 seconds). No significant correlation existed between plasma fibrinogen, prothrombin time and platelet aggregation in normal BMI or low BMI groups. Antithrombin IIIactivity in normal BMI group was $108 \%$ and in low BMI group it was $105 \%$. Atendency of positive correlation existed between antithrombin III activity and BMI in low BMI pill users $(r=0.591, p=0.072)$.

Conclusion:The study suggested that a) Reported risk of procoagulant or thromboembolic changes in pill users is lower in low BMI \&normal BMI and b) Low BMI users showed significantly longer prothrombin time due to the effect of malnutrition itself or due to the effect of pills in this nutritional background.

Keywords: Low dose OCP, Coagulation status, Normal and low BMI women.

(J Bangladesh Coll Phys Surg 2017; 35: 3-8)

contraception and is well accepted by women ofvarious socioeconomic conditions. Millions of women use these drugs. So questions regarding the safety of these agents are important.

A number of side effects were encountered by the users. These included nausea, vomiting, dizziness, metabolic disorders such as hypertention, diabetes and thromboembolic manifestations in the form of pulmonary embolism, leg vein thrombosis, coronary thrombosis and thus the estrogen component of the OCP was initially implicatedin the pill-induced thromboembolic side effects $^{1,2}$. But later it was shown that the gestagen componenttoo played a vital role in enhancing cardiovascular side effects ${ }^{3,4}$. The introduction of low dose OCP with $30 \mu \mathrm{g}$ estrogens was a step in the right direction but further reduction resulted in breakthrough bleeding ${ }^{5}$. The Dunlop committee for the safety of drugs in UK issued a declaration linking the estrogenic component 
of the pill with the risk of venous thrombosis. Consequently the doses of estrogen in the pill were reduced again and again until affecting contraceptive efficacy. As a result, the risks which were attributed to high estrogen content such as venous thrombosis, hypertension, diabetes etc were reduced. This was confirmed by the Royal College of general practitioners and the Walnat Creek Contraceptive drug study ${ }^{6}$.

However, such low doses was possible primarily because highly effective and more specific progestogen such as levonorgestrel, desogestrel, gestadene, norgestimateetc were developed and introduced. The development of Levonorgestrel and third generation progestogens were potentially responsible for reducing severe cardiovascular side effects ${ }^{7}$.

Several retrospective and prospective studies have suggested the existence of an increased risk of cardiovascular diseases, in particular venous thromboembolism, pulmonary embolism, myocardial infarction (MI) and hemorrhagic stroke in users of OCP a risk possibly related to the dose of estrogen ${ }^{8,9,10}$. It has been suggested that adhesiveness of blood platelets to a glass surface was greater in women than in man in the presence of a histone like substance derived from human brain, the adhesiveness of platelets were increased in woman because of OCP therapy ${ }^{11}$.

A comparisonof Japanese and American women using OCP, concluded that the effect on the fibrinolytic system were considerably better among the Japanese women than among the Americans, indicating a decreased risk of cerebral thrombosis in Japanese women ${ }^{12}$.

Antithrombin III (AT III) activity fell significantly during OCP useand the estrogen component was blamed for the fall ${ }^{13,14}$. Low serum ATIII activity was observed in patients with pulmonary embolism,MI and venous thrombosis. The use of low estrogen pill resulted in a significant reduction in the number of thromboembolic episodes and the reduction was found to be limited in venous thromboembolism ${ }^{15}$.

Effect of OCP on Caucasian woman was studied and observed acceleration of the procoagulants in extrinsic and intrinsic clotting systems and reduction of the plasma AT III levels, indicating some imbalance of the hemostatic mechanism towards hypercoagulability. These changes increased with the duration of therapy and took some months to resolve when OCP were withdrawn. OCP should be avoided by woman with established cardiovascular disease or with cardiovascular risk factors, irrespective of age ${ }^{16}$. They further suggested that OCP appear to unsuitable method of contraception even for healthy and nonsmoker women over 40 years of age.

A large number of Bangladeshi women of various socioeconomic status are using low dose OCP. There are different types of combined low dose OCP used by the Bangladeshi women eg: Shukhi ${ }^{(\mathrm{R})}, \mathrm{Femicon}^{(\mathrm{R})}$, Nordette- $28^{(\mathrm{R})}$ and Marvelon ${ }^{(\mathrm{R})}$. All these OCPs contain $30 \mu \mathrm{g}$ of ethinyl estradiol, but their progestogen component varies.

Shukhi is mostly used by the women of low socioeconomic status, because the family planning program of the government of Bangladesh has made it available to them free of cost. Studiesshows that newer combined low dose OCPs cause less upset in metabolic and coagulation parameters as it contains $30 \mu \mathrm{g}$ of ethinyl estradiol (EE) $)^{17,18}$. But these need to be confirmed in Bangladeshi population with low BMI as it is well known that diet and life style can affect pill induced changes in hepatic protein synthesis. Safety of OCP has not been studied in low BMI undernourished Bangladeshi women.Due to protein deficiencies in these low BMI groups there may be different biological response in these women resulting in different risk profile.

\section{Materials and Method:}

The study was conducted at the Department of Cell and Molecular Biology, Research Division, Bangladesh Institute of Research and Rehabilitation in Diabetes, endocrine and Metabolic disorders (BIRDEM) during the period of Jan2001 to Dec 2001. The patients were collected from family planning centre, Dhaka Medical college Hospital (DMCH). A total number of 40 women were included in this study having age range between 25-45 years. 29 were with normal BMI (>18.5) and 11 were with low BMI $(<18.5)$ consuming Shukhi for six months to five years. Patients who were normotensive with no coexisting medical disorders were included. Patients who were hypertensive, diabetic, smoker, alcoholic and obese (BMI $>30)$ were excluded.

Subjects: After taking consent of the patient blood samples were collected and plasma/serum were stored at $-70 \mathrm{C}$ for biochemical analysis. The coagulation factorswere assessed as follows: Coagulation status- 
(P Fibrinogen, Prothrombin time and Platlet aggregation) and anticoagulation status -Antithrombin III (ATIII).

Laboratory methods: Plasma fibrinogenwas estimatedby clotting method; Prothrombin time by using SimplastinExcel; Platelet aggregation by optical aggregometry and Antithrombin III was estimated by amidolytic method using a synthetic chromogenic substrate.

All statistical analysis were done by using SPSS. Results were expressed as median (range). The statistical comparison between the groups were done by MannWhitney test. Difference betweenBMI and other parameters were analysed by Pearson's correlation coefficient test. $\mathrm{P}$ value $<0.05$ was taken as level of significance.

\section{Different groups discussed are as follows:}

1. Clinical features (Age, BMI, Duration of pill use) of Normal BMI and low BMI study subjects.

2. Coagulation status (eg: Platelet aggregation, Plasma fibrinogen and Prothrombin time)of Normal BMI and low BMI study subjects.

3. Coagulation status among the Normal BMI andlow BMI study subjects with the same duration of OCP use ( $<24$ months).

4. Antithrombin III activity of Normal BMI and low BMI study subjects.

5. Pearson correlation coefficient between BMI and coagulation status (Platelet aggregation, Fibrinogen, Prothrombin time) and Antithrombin III.
6. Pearson correlation coefficient between plasma fibrinogen and Antithrombin III in Normal and Low BMI Group.

\section{Results:}

1.Clinical features of different groups of the study subjects shown inTable I. No significant difference was observed in clinical features of different groups.

2.Coagulation status-Platelet aggregation, Plasma fibrinogen, Prothrombin time of study subjects. There was no statistically significant difference in platelet aggregation $(\mathrm{p}=0.387)$ and plasma fibrinogen level ( $p=0.332$ ) between the Normal BMI and Low BMI groups studied .

3. There is significant difference in prothrombin time between the two groups $(p=0.019)$, and significantly prolonged prothrombin time was noted among the Low BMI pill users (Table-III)

4.Antithrombin III activity of the study subjects. There was no statistically significant difference found in plasma antithrombin III activity between Normal and Low BMI groups ( $\mathrm{p}=0.421)$, showed in table-IV.The AT III activity has found to gone up towards the upper limit in both groups of pill users (normal value 88-111\%).

5.Pearson correlation coefficient between BMI and coagulationstatus and ATIII. A significantly towards positive correlation was found among AT III and Low $\operatorname{BMI}(\mathrm{r}=0.591, \mathrm{p}=0.072)$ No significant correlation was found among others with BMI

6. A positive correlation was found between fibrinogen and antithrombin III in normal BMI, which is not maintained in Low BMI

Table-I

\begin{tabular}{lcc}
\multicolumn{3}{c}{ Clinical features of different groups of study subjects } \\
Groups & Normal BMI $(\mathrm{n}=29)$ & Low BMI $(\mathrm{n}=11)$ \\
\hline Age (years) & $28.00(25-45)$ & $26.00(25-32)$ \\
BMI (kg/m2) & $23.06(19.40-30.08)$ & $18.44(15.04-18.50)$ \\
Duration (months) & $24.00(7-60)$ & $12.00(6-24)$ \\
\hline
\end{tabular}

Results are expressed as median (range)

Normal BMI=Subjects with normal BMI

Low BMI=Subjects with low BMI

$\mathrm{n}=$ Number of subjects

BMI=Body Mass Index

Duration $=$ Duration of OCP use 
Table-II

\begin{tabular}{lccc}
\multicolumn{4}{c}{ Coagulation status of Normal BMI and low BMI study subjects. } \\
Groups & Normal BMI $(\mathrm{n}=29)$ & Low BMI $(\mathrm{n}=11)$ & $\mathrm{U} / \mathrm{p}$ value \\
\hline Platelet aggregation(\%) & $20(6.25-71.25)$ & $20(6.25-47.50)$ & $131 / 0.387$ \\
Plasma fibrinogen(mg/dl) & $450(165-700)$ & $318(142-788)$ & $127.50 / 0.332$ \\
Prothrombin time(Second) & $12.60(10.50-16)$ & $13.80(12.00-16.30)$ & $97.00 / 0.058$ \\
\hline
\end{tabular}

Table-III

Coagulation status among the Normal BMI and low BMI study subjects with the same duration of OCP use ( $<24$ months).

\begin{tabular}{lccr} 
Groups & Normal BMI $(\mathrm{n}=20)$ & Low BMI $(\mathrm{n}=11)$ & U/p value \\
& use OCP $<24$ months & use OCP $<24$ months & \\
\hline Platelet aggregation(\%) & $21.88(6.25-71.25)$ & $20(6.25-47.50)$ & $88.50 / 0.373$ \\
Plasma fibrinogen $(\mathrm{mg} / \mathrm{dl})$ & $452(165-700)$ & $318(142-788)$ & $83.50 / 0.274$ \\
Prothrombin time(second) & $12.20(11.10-16.00)$ & $13.80(12.00-16.30)$ & $53.50 / 0.019$ \\
\hline
\end{tabular}

\section{Table-IV}

Antithrombin III activity of Normal BMI and low BMI study subjects.

\begin{tabular}{lccr} 
Groups & Normal BMI $(\mathrm{n}=29)$ & Low BMI $(\mathrm{n}=11)$ & U/p value \\
\hline Antithrombin III $(\%)$ & $108(66-210)$ & $105(76-134)$ & $120.00 / 0.421)$ \\
\hline
\end{tabular}

Table-V

Pearson correlation coefficient between BMI and coagulation status and Antithrombin III.

\begin{tabular}{|c|c|c|c|c|}
\hline \multirow[t]{2}{*}{ Group } & \multicolumn{2}{|c|}{ Normal BMI } & \multicolumn{2}{|c|}{ Low BMI } \\
\hline & $\mathrm{r}$ & $\mathrm{P}$ & $\mathrm{r}$ & $\mathrm{P}$ \\
\hline Pl.agg & \multicolumn{2}{|c|}{098.614} & \multicolumn{2}{|c|}{$-.500 .117$} \\
\hline Fibrinogen & -.033 & .867 & -.483 & .132 \\
\hline PT & .036 & .855 & .189 & 577 \\
\hline AT III & -.033 & .865 & .591 & 072 \\
\hline
\end{tabular}

Pl.agg $=$ Platelet aggeregation, $\mathrm{PT}=$ Prothrombin time, ATIII=Antithrombin III.

Table-VI

\section{Pearson correlation coefficient between plasma fibrinogen and Antithrombin III} in Normal and Low BMI Group.

\begin{tabular}{llcll}
\multirow{2}{*}{ Groups } & \multicolumn{2}{c}{ Normal BMI $(\mathrm{n}=11)$} & & \multicolumn{2}{c}{ Low BMI $(\mathrm{n}=11)$} \\
\cline { 2 - 3 } & $\mathrm{r}$ & $\mathrm{P}$ & $\mathrm{r}$ & $\mathrm{P}$ \\
\hline Fibrinogen/AT III & .355 & .059 & -.189 & .602 \\
\hline
\end{tabular}




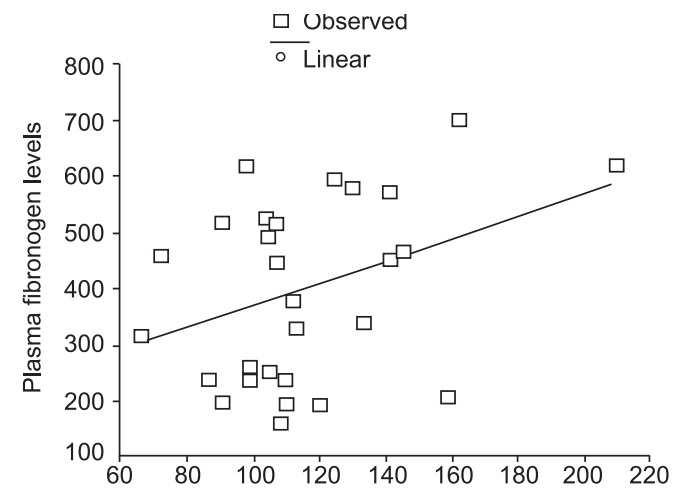

Plasma anti-thrombin III of the subjects having normal BMI

Fig.-1: Person correlation coefficient between plasma fibrinogen and plasma antithrombin III of normal BMI OCP users.

\section{Discussion:}

Subtle but complex changes occur in coagulation mechanism in women on OCP. Oral contraceptives have been shown to induce more rapid platelet aggregation and adhesion and also to reduce the activity of the fibrinolytic system, which normally breaks down the blood clot. Thus, when clot forms, they may be less likely to dissolve and more likely to reach a size sufficient to block vessels and create a thromboembolic problem. The increased risk of venous thrombosis and pulmonary embolism is probably attributable in some women to the estrogencontent of their oral contraceptives. A positive correlation was found by Inman et al in United Kingdom between the risk of thromboembolic disease and the dosage of estrogen contained in $\mathrm{OCP}$, when combined OCP containing high doses (75-100 ì or more) of estrogen, were available at that time were used ${ }^{19}$. The availability of new generation monophagic and multiphagic formulations of OCP containing both low dose of estrogen and new progestogen has virtually changed the previous idea of thrombogenic effect of $\mathrm{OCP}^{20,21}$.

The general belief is that OCP intake leads to a high index of suspicion of thromboembolic episodes of pill users.There is limited data available regarding thrombogenic potential of OCP among Bangladeshi users. In this study, the effects of OCP on certain parameters were measured.To see the coagulation status platelet aggregation, plasma fibrinogen, prothrombin time and antithrombin III activity were measured. There was no significant change in platelet aggregation, plasma fibrinogen and antithrombin III activity in the two groups of OCP users. The prothrombin time in low BMI users was slightly prolonged by 1.6 second in contrast to normal BMI (Table III), which was statistically significant.There may be deficiency in ionized calcium in low BMI OCP users as the study sample are from poor socioeconomic status. The plasma fibrinogen in normal BMI subject has gone up above the upper range as compared to low BMI subject (450mg/dl Vs $318 \mathrm{mg} /$ $\mathrm{dl}$ in Normal BMI Vs Low BMI) but the difference is not significant $(\mathrm{p}=0.332)$. In this study ATIII activity in both groups were gone at the upper limit eg: in normal BMI it is $108 \%$ and in low BMI 105\% (normal range : 88 $111 \%)$. It is well known that OCP increases the plasma fibrinogen level. Plasma fibrinogen and AT III activity in Normal BMI subjects has gone above the upper range ( $450 \mathrm{mg} / \mathrm{dl}$ and $108 \%$ in Plasma fibrinogen and ATIII). A positive correlation found between plasma fibrinogen and AT III in the normal BMI OCP users $(r=0.355, p=0.059)$. We can say from the above relationship between plasma fibrinogen and AT IIIthat the rise of plasma fibrinogen is due to pill use and rise in ATIII is a secondary response to plasma fibrinogen to counterbalance the coagulation activity. It may be assumed that after a certain level of BMI increment the plasma fibrinogen will be so high at which the ATIII will not be able to counterbalance with prolong use of OCP.There was no correlation exists between the plasma fibrinogen and AT III of Low BMI OCP users. There is no significant difference in platelet aggregation in normal BMI and low BMI groups. David et al revealed in their study that the platelet count, platelet aggregation ratio were not significantly altered and antithrombin III activity was not reduced among the users of low dose oral contraceptives $^{20}$.

Sinceprolonged prothrombin time was found within low BMI OCP users and a positive correlation existed between AT III and low BMI subjects. Reasons for such findings may be nutritional or OCP in this nutritional background. So a large extensive and indepth further study with prothrombin time, AT III, serum calcium, total plasma protein and serum albumin are also required to clarify this issue in nutritionally deprived low BMI OCP users.

Finally, the less number of low BMI subject is a limitation of this study. Initially it was thought that number of low 
BMI women of child bearing age using OCP will be widely prevalent. During data collection it was found that low BMI women in this age group are not so common as compare to normal BMI. Cause may be gaining of weight and less linear height in this nutritional group. Reasons for gaining of weight of these women might be child bearing, increasing age or OCP itself. On the otherhand, average height of Bangladeshi women are low in comparison to nutritionally privileged women possibly this is how the normal range of BMI is maintained in women with low socioeconomic background and also the BMI parameter in developing country is different from that of developed country.

\section{Conclusion:}

From the statistical analysis of the results obtained in present study and their comparison data suggested the following:

Low dose OCP do not seem to affect the coagulation status of Low BMI Subjects. The reported risks ofprocoagulant or thrombogenic changes in pill usersseem to be dependent on obesity, in the lower weight playing a protective role. Precautions, however, is necessary for possible bleeding disorders in low BMI users who showsignificantly longer prothrombin time due to the effect of malnutrition itself or due to the effect of pills in this nutritional background. Further studies are also required to clarify this issue of prothrombin time. AT III levels are not probably affected in underweight OCP users.It probably changes proportionately with serum fibrinogen in Normal BMISubjects. But this relationship can not be maintained in Low BMI Subjects.

\section{References:}

1. Royal College of General Practioners. Oral Contraceptives and health. An interim report for the oral contraception study of the Royal college of general practitioners. New York, Pitman Publishing Co. 1974; 98.

2. Prasad RNV, Ratnam SS. The cardiovascular and thromboembolic risks of oral contraception-A review. Sing J. ObstelGynecol 1980; 11:1, pp 7-19.

3. Ratnam SS,Prasad RNV. Oral contraceptives In: Practice of Fertility control (Chaudhuri,SK, Ed) Current Book Publishers, Calcutta 1983; p 103.

4. Ulysse JG Metabolic effects of Oral contraceptives. Am. J. ObstelGynecolpt II suppl, 1987;157:4, 1029-1014.

5. Ratnam SS,Prasad RNV. Recent developments in steroidal contraception. Sing J. Obstel Gynecol. 1980; 11:pp 7-13.
6. Hussain's current status of oral contraceptives. Bangladesh J.physiol. And Pharmacol. June 199; 7(1):40-41.

7. Skouby SO, Peterson KR and Jespersen J. The influence of new dose oral contraceptive on metabolic variables.Adv.Contra.Dec 1991; 7(suppl.2):77-88).

8. Start well PE, Masi AT, Arthes FG, Green GR and Smith HE. Thromboembolism and oral contraceptive. An epidemiologic Case Control Study. Am.J. Epidem. 1969;90:365-380.

9. Stadel BV. Oral contraceptive and cardiovascular disease. N. Engl. J. Med. 1981 a: 305(11): 612-618.

10. Anonymous. Oral contraceptives: mechanism in thromboembolism. Lancet. May 1980; 1118-1119.

11. Caspray EA and Peberdy M. Oral contraceptive and blood platelet adhesiveness Lancet, May 1965; 1142-1143.

12. Ogino M. Effect ofOral contraceptives on fibrinolytic system among Japanese and American women. Nippon SankaFujinkaGakkaiZasshi, ActaObstetrica EtGynocologica japonica. June 1986; 38(6): 817-826.

13. Vonkaulla E andVonkaulla KN. Oral contraceptives and low antithrombin III activity. Jan 1970; Lancet 1, 36.

14. Howei PW, Prentice CRM, Mallinson AC, Horne CHW and McNicol GP. Effect of combined estrogenprogesteron oral contraceptives, estrogen and progestogen on antiplasmin and antithrombin activity. Dec 1970; Lancet: $1329-1332$.

15. Bottiger LE, Boman G, Eklund G and Westerholm B. Oral contraceptives and thromboembolic disease: Effects of lowering estrogen content. Lancet, May 1980; 1027-1101.

16. Poller L. Oral contraceptives, blood clotting and thrombosis. Br. Med. Bull. 1978; 34(2): 151-156.

17. Samsioe G. Comparative effects of Oral contraceptive combinations $0.15 \mathrm{mg}$ desogestrel $+0.03 \mathrm{mg}$ ethinyloestradiol and $0.15 \mathrm{mg}$ levonorgestrel $+0.03 \mathrm{mg}$ ethinyloestradiol on lipid and lipoprotein metabolism in healthy female volunteers. Contraception 1982; 25: 5, 487-503.

18. Lanchnit- Fixon. Triphasic pill "neutral"effects on lipid profile.XI world congress on fertility and sterility Newsletter. Schering AG.Publishers 1983;4.

19. Inman WHW, Vessey MP, Westerholm, Engeland A. Thromboembolic disease and the steroidal content of oral contraceptives. A report to the committee on safety of drugs. BMJ. April 1970; 2:203-209.

20. David JL. Gaspard UJ. Gillain D, Raskinet R, Leport MR. Haemostasis profile in woman taking low dose oral contraceptives. Am. J. Obstel. Gynecol.July 1990; 163 (1): 420-423. 